\title{
Photo-microdomains in ferroelectrics: formation and light scattering caused by them
}

\author{
A.N. Morozovska ${ }^{1}$, E.A. Eliseev ${ }^{2}$, V.V. Obukhovsky ${ }^{1}$, V.V. Lemeshko ${ }^{1}$ \\ ${ }^{\prime}$ Taras Shevchenko Kyiv University, Radiophysic Department, 64 Volodymyrs'ka str., 01033 Kyiv, Ukraine \\ e-mail:morozo@mail.i.com.ua,obukhovsky@univ.kiev.ua \\ ${ }^{2}$ Institute for Materials Sciences, NAS of Ukraine, 3 Krjijanovskogo, 252180 Kiev, Ukraine
}

\begin{abstract}
We propose the theory of the micro-domains (MD) formation in ferroelectric photorefractive crystals appeared under steady illumination by laser beam perpendicular to the polar axis.

The crystal has the donor level made of photoactive impurity atoms. The longitudinal photovoltaic current leads to surface charges accumulation at the light spot boundary. These charges are localized at the nano-clusters of different size and charge density randomly distributed in the thin transition layer between light and dark. Each such cluster can be treated as the seeding for one MD growth. Therefore the numerous MD appear around the illuminated area. The micro-domain shape and physical properties are studied in the phenomenological GinsburgLandau-Devonshire theory framework with respect to the screening effects of ferroelectric medium.

We obtained, that when the cluster charge density is more that the critical one, MD become very long and thin ones and in principle can intergrow through the perfect sample. In such a case they could be easily registered experimentally. So, exactly due to the transverse modulation effects the MD length can be sharply increased from the dozens of microns and up to the crystal length.

All theoretical results are in a good qualitative agreement with our experiments on photomicro-domain formation in $\mathrm{LiNbO}_{3}$ crystals and light scattering caused by them.
\end{abstract}

Keywords: micro-domains, photorefractive ferroelectric crystals, GLD-theory, screening effects.

Paper received 27.05.03; accepted for publication 17.06.03.

\section{Introduction}

Nowadays the problems of light-induced micro-domain (MD) formation in the different ferroelectric materials are of especial interest owing to their potential usage in optical information storage. So, both experimental data and theoretical investigations concerning generation conditions, stability and properties of MD are extremely desirable.

Let us consider the process of the micro-domain (MD) onset [1] in the uniaxial ferroelectric near the charged volume defects (clusters) of the different nature. There exist both shallow donor levels and deep traps being able to generate or to capture free charge carriers in photorefractive crystals (PRC). Clusters arisen due to implementation of the non stechiometric (i.e. with the small solubility limit) photoactive impurity will be of our essential interest [2]. Really, such impurity atoms tend to cluster-like formation with size about $(0.5 \div 5) \mathrm{nm}$ (see e.g.
Ref. [3]). Under the light illumination clusters become positively charged owing to photocarriers transfer to the conduction band or negatively charged due to the photocarriers capture. The illuminated region has much larger conductivity owing to photocarriers than the not illuminated one. Owing to this fact and photovoltaic effect [4], photoinduced inner field is rather high and strongly influences on the spontaneous induction amplitude and distribution inside the light area. So, in the case of abrupt light and dark boundary, the spontaneous induction screening effects outside the illuminated area must be taken into account. Moreover, one can assume that screening space charges, localized in the very thin transition layer at the light and dark boundary in the form of charged clusters, could cause the MD formation process. Hereinafter we regard this screening charge as surface charge with spatially distributed density.

We will show, that these screening surface charges will lead to the surrounding repolarization and the nu- 


\section{A.N. Morozovska et al.: Photo-microdomains in ferroelectrics: formation ...}

merous needle-like micro-domains arise outside the illuminated area. Under definite values of material parameters MD longitudinal size substantially exceeds the transversal one and they grow through the sample [5].

\section{The model}

Now we investigate the question about the initial mono domain PRC spontaneous induction $\mathbf{D}$ changes with the appearance of the illuminated area with the longitudinal size $a_{0}$ and inhomogeneous surface charge density with the average transverse period $b_{0}$. The calculations scheme is represented in Fig. 1. The spontaneous induction is aligned along $z$ axis far from the cluster. Light beam has the cylindrical form with axis parallel to $y$ axis. Hereafter we suppose that $b_{0}<<a_{0}$ and the boundary between light and dark is rather sharp.

Charged clusters localized at the light spot edge create the additional surface charge with density $\sigma(x, y, z)$ (we estimate it in 3.2). This density reaches its maximal value at the points with the maximal photoactive impurity concentration. Therefore maximal electric field is produced near these points and with the appropriate charge sign it will lead to the surrounding repolarization around the illuminated area. Note that it is not worth to say about repolarization inside the cluster because ferroelectric phase is absent due to the local symmetry breaking in this region.

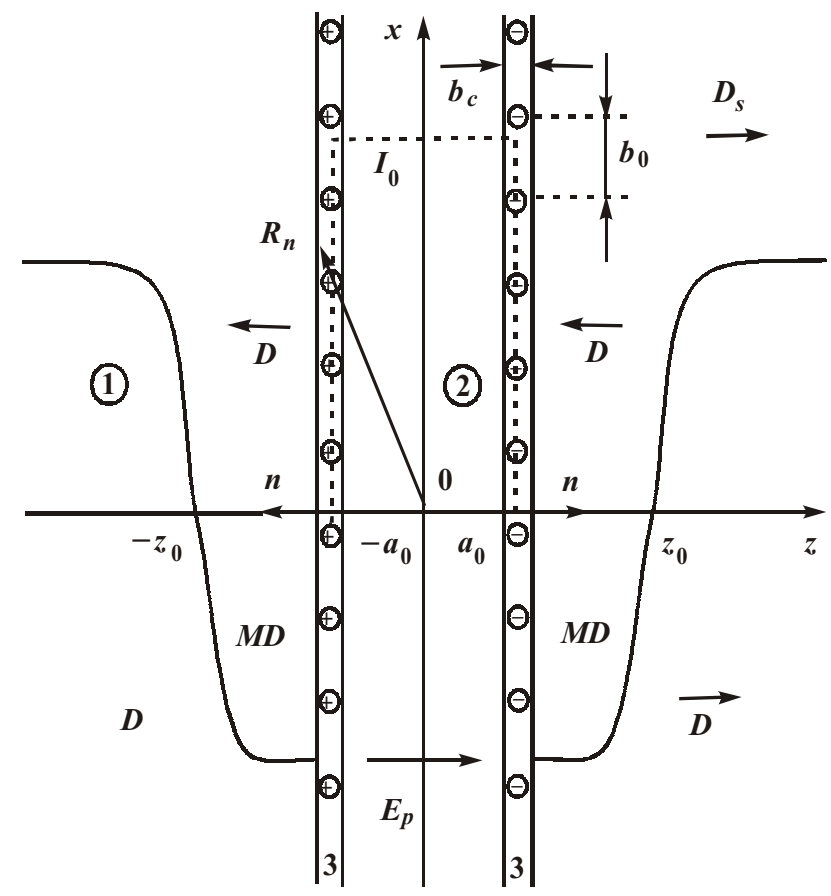

Fig. 1. The calculations scheme with the spontaneous induction distribution (solid line in region "1") outside illuminated area, denoted by " 2 ". Dashed line represents the light intensity distribution inside the illuminated area " 2 " and the transition layer " 3 ".

SQO, 6(3), 2003

\section{General equations}

\subsection{Stationary equations outside the light area}

The stationary distribution of the induction outside the light area is described by the following system of equations [6], namely, phenomenological equation of state for second order phase transitions:

$\alpha_{T} D+\beta D^{3}-\gamma \Delta D=E$,

where $\alpha_{T}=\alpha\left(T-T_{c}\right)<0, T_{c}$ is the phase transition temperature, $E$ is the electric field, which is sum of the internal (depolarization) and external field. Hereafter we consider the case without the external electric field, i.e. $E$ makes sense of the internal field. Here we suppose that the absolute value of longitudinal induction component $D_{z}$ is much larger than the transversal ones $D_{x, y}$ and denote $D_{z}=D$ (see commentary to (17)).

Note that contribution of homogeneous elastic strain to the equation ( $1 \mathrm{a})$ is reduced to the renormalization of the coefficients $\alpha_{T}$ and $\beta$ (see, e.g. Ref. [7]), but it is known that the inhomogeneous distributions of the spontaneous induction like domain walls lead to the inhomogeneous distribution of the elastic strain [8]. These kinds of structures require support of inhomogeneous mechanical constraints, like defects and dislocations. In order to take these effects into account it is necessary to add to (1a) electrostriction terms and take into consideration the elastic equilibrium conditions. Hereafter we neglect the inhomogeneous strain presence. The more complicated models considering inhomogeneous strain influence is are progress now.

Maxwell equations and the condition of macroscopic current absence have to be added to (1a):

$$
\begin{gathered}
\frac{d D}{d z}=4 \pi e \rho, \quad \rho=\rho_{h}-\rho_{e}, \quad q_{h}=-q_{e}=e \\
\mathbf{j}=\sum_{k=h, e}\left[-\delta_{k} \nabla \rho_{k}+\mu_{k} \rho_{k} \mathbf{E}\right] q_{k}=0, \quad \mathbf{E}=-\nabla \varphi,
\end{gathered}
$$

where $\varphi$ is the electric potential, $\rho_{h, e}$ is the proper (dark) bulk charge density of holes and electrons respectively, $\delta_{h, e}, \mu_{h, e}$ are their diffusion coefficient and mobility, $e$ is the absolute value of electron charge. Here we consider the diffusion and the conductivity currents. Taking into account Einstein relation $q_{k} \delta_{k} / \mu_{k}=k_{B} T$, it is easy to obtain from (1b) that outside the illuminated area bulk charge density $\rho$ has the form

$\rho=-\rho_{0} \sinh \left(\frac{e \varphi}{k_{B} T}\right) \quad \rho_{h}^{0}=\rho_{e}^{0}=\frac{\rho_{0}}{2}$,

where constants $\rho_{h, e}^{0}$ are dark charge densities, which do not depend on light intensity, existing only in the illuminated area. System (1) must be supplemented with the boundary conditions

$D\left(|\mathbf{r}|>>\left|\mathbf{R}_{n}\right|\right)=D_{s},\left.\left(D_{n}-D_{c n}\right)\right|_{\mathbf{r}=\mathbf{R} n}=4 \pi \sigma$ 


\section{A.N. Morozovska et al.: Photo-microdomains in ferroelectrics: formation ...}

Here vector $\mathbf{R}_{n}$ determines the illuminated area surface, $\mathbf{n}$ is its outer normal (see Fig. 1), $D_{s}$ is spontaneous induction far from the light spot, in the bulk ferroelectric it equals to $\pm \sqrt{-\alpha_{T} / \beta}, D_{c n}$ is induction exactly inside the light spot edge Representation (2) corresponds to the mechanism of the free carriers thermalization [4] in contrast to $\sigma$ which represents the surface density of the charge localized in the deep levels.

Differentiation of the first equation (1b) with respect to the second one and equation (2) leads to the following relationship:

$$
E=R_{D}^{2} \sec h\left(\frac{e \varphi}{k_{B} T}\right) \frac{d^{2} D}{d z^{2}}, \quad R_{D}=\sqrt{\frac{k_{B} T}{4 \pi e^{2} \rho_{0}}},
$$

where $R_{D}$ is the Debye-Hukkel screening radius.

In the dark region of the sample far from the outer boundaries (regions 1) we suppose that $|e \varphi|<<k_{B} T$. So $\sec h\left(e \varphi / k_{B} T\right) \rightarrow 1$, one can essentially simplify equation (4) and express electric field $E$ via the second derivative of the induction $D$ only:

$E \approx R_{D}^{2} \frac{d^{2} D}{d z^{2}}$

Contrary, exactly on the light spot boundary (transition layer 3) we suppose that $|e \varphi| \gg>k_{B} T$. So $\operatorname{sech}\left(e \varphi / k_{B} T\right) \rightarrow 0$. If the light intensity decreases monotonically approaching the spot edge, the second derivative of the induction $D$ is finite, electric field $E$ exponentially decreases:

$E \approx 0$.

Using the relation (5a) it is easy to rewrite equation (1a) outside light spot with renormalized correlation energy

$$
\alpha_{T} D+\beta D^{3}-\left(\gamma\left(\frac{d^{2}}{d x^{2}}+\frac{d^{2}}{d y^{2}}\right)+\left(\gamma+R_{D}^{2}\right) \frac{d^{2}}{d z^{2}}\right) D=0 .
$$

Note, that this equation is homogeneous in contrast to the inhomogeneous equation (1a). Estimations of $R_{D}$ and $\gamma$ values have the view $\gamma \sim 10^{-14} \div 10^{-16} \mathrm{~cm}^{2}, R^{2}{ }_{D} \sim$ $3 \cdot\left(10^{-13} \div 10^{-11}\right) \mathrm{cm}^{2}$. It is seen that $R^{2}{ }_{D}>>\gamma$ and equation (6) is substantially anisotropic:

$\gamma_{L}=\gamma+R_{D}^{2}>>\gamma_{x, y}$

Free energy density has the following form

$g=\frac{\alpha_{T}}{2} D^{2}+\frac{\beta}{4} D^{4}+\frac{\gamma}{2}\left(\frac{d D}{d x}\right)^{2}+\frac{\gamma}{2}\left(\frac{d D}{d y}\right)^{2}+\frac{\gamma_{L}}{2}\left(\frac{d D}{d z}\right)^{2}$

Hereinafter, in order to obtain the distribution of $D$ one could solve either (6) or minimize free energy (8), taking into account the boundary conditions (3).

\subsection{Stationary solutions inside the light area}

Let us consider the symmetrical with respect to $x$ axis illuminated area $|z|<a_{0}$ with constant light intensity $I_{0}$, centered in the coordinate origin (region 2 in Fig. 1). Under the sufficient intensity and for the light quanta energy higher than the threshold one, the photoconductivity inside illuminated area is much greater then the dark one, i.e. the latter can be neglected. Really the free carriers density in the illuminated area is much larger than the one outside it. Owing to this fact equation of state (1a) is valid inside the illuminated area 2 , but with essentially renormalized coefficients $\alpha_{T}, \beta, \gamma$ [4]. This renormalization and high values of photovoltaic inner field $E_{p} \sim(30 \div 80) \mathrm{kV} / \mathrm{cm}$ (see Ref. [9]) (really $a_{0} E_{p}>>$ $>>k_{B} T / e$ ) strongly influence on the spontaneous induction amplitude and distribution inside the light area. So, in the case of abrupt light and dark boundary, the spontaneous induction screening effects outside the illuminated area must be taken into account. Moreover, one can assume that screening space charges, localized at the boundary in the form of charged clusters can cause the MD formation process.

Thus, when neglecting the dark conductivity $\rho_{h, e}<<n_{e}$, the inner electric field $E_{p}$ inside the illuminated area (region 2) can be found from the Maxwell equations and the condition of macroscopic current absence [10]:

$$
\frac{\partial n_{d}^{+}}{\partial t}=-\Gamma n_{e} n_{d}^{+}+s I_{0}\left(n_{d}^{0}-n_{d}^{+}\right)
$$

$\frac{\partial n_{e}}{\partial t}=\frac{\partial n_{d}^{+}}{\partial t}+\frac{d i v \mathbf{j}_{n}}{e}$

$\operatorname{div} \mathbf{D}=4 \pi e\left(n_{d}^{+}-n_{e}\right)$,

$\mathbf{j}_{n}=e \mu_{e} n_{e} \mathbf{E}_{p}+e \delta_{e} \nabla n_{e}+\left(n_{d}^{0}-n_{d}^{+}\right) s I_{0} \mathbf{G}$

Where the following designations are introduced: $n_{e}$ is the concentration of free electrons, $n_{d}^{0}$ is the concentration of donors, $n_{d}^{+}$is the concentration of ionized donors (traps), $\Gamma$ is the capture coefficient of an electron by a trap, $\mathrm{s}$ is the photoionization coefficient, $I_{0}$ is the pump intensity, e is the absolute value of the electron charge, $\mathbf{j}_{n}$ is the density of electron current, $\mu_{e}$ is the electron mobility, $\delta_{e}$ is the diffusion coefficient, $G_{k}=\beta_{k l m} e_{l} e_{m}^{*}$ is the Glass vector components (the double convolution of the photovoltaic-tensor with the pump wave polarization $e_{p}$ ). For the materials with $\left|G_{z}\right|>>\left|G_{x, y}\right|$ the transverse currents are the same order that the dark ones. So let us consider only the influence of longitudinal photovoltaic current, i.e. $G \equiv G_{z}$ and $E_{p} \approx E_{p z}$.

After elementary transformations and standard bifurcation analyses [11], one can show that time-independent solution of nonlinear system (9) with constant coef- 


\section{A.N. Morozovska et al.: Photo-microdomains in ferroelectrics: formation ...}

ficients $n_{d}^{0}, \mu_{e}, \delta_{e}, G_{z}$ and equation of state (1a) with renormalized coefficients due to the free carriers presence [4] is stable in the vicinity of the stable stationary point $\mathbf{j}=0, \operatorname{div} D=0, \alpha_{T} D+\beta D^{3}=E_{p}$. This solution is the following:

$E_{p}=-\frac{G_{p z} \Gamma}{e \mu_{e} s} n_{d}^{+}, \quad n_{e}=n_{d}^{+}=\sqrt{\left(\frac{s I_{0}}{2 \Gamma}\right)^{2}+\frac{s I_{0} n_{d}^{0}}{\Gamma}}-\frac{s I_{0}}{2 \Gamma}$,

$\alpha_{T} D+\beta D^{3}=E_{p}$

Note that below transition temperature the absolute value of coefficient $\alpha_{T}<0$ is much smaller than unity. As it follows from (9b), always $n_{d}^{0} \geq n_{e}$, as it should be expected. Note, that $G_{p z} \sim D_{s}$, [4]. It is seen that electric field $E_{p}$ is proportional to induction $D_{s}$. If they have opposite signs, $E_{p}$ can significantly decrease $D_{s}$ and can be regarded as depolarization field.

It is worth to underline, that (9b) is valid only in region 2 (see Fig. 1), but not inside the transition layer 3, where $E_{p}$ decreases to zero due to both $I_{0} \rightarrow 0$ and $|e \varphi|>>k_{B} T$ (see (5b)). Really, in the transition layer the real $I_{0}$ and $D$ gradients could not be neglected. These gradients and inhomogeneous distributions of photoactive clusters with localized space charge in the region can cause the local symmetry breaking and spontaneous induction destroying from $D=D_{s}$ at the transition layer inner boundary 23 to $D_{s} \rightarrow 0$ and $E_{p}=0$ at the transition layer outer boundary 31 . In other words transition layer 3 can be regarded as screening layer with usual screening mechanism by space charge layer [6], [12].

Thus, how can the field determining by (9b) be compensated outside the light area in accordance with $(5 \mathrm{~b})$ ? This field is compensated by the free carriers, moved by the field inside the transition layer and captured by the deep traps on the illuminated area boundary. This process lasts up to the moment when they generate the surface charge density enough to decrease the field to zero. Charged photo carriers, absorbed by the deep traps at the abrupt spot edge boundary, create the additional surface charge with density $\sigma(x, y, z)$ stably localized at the photoinduced inhomogeneities (charged clusters) on light spot edge. Let us estimate the effective value of $\sigma(x, y, z)$.

We assume that screening transition layer formation almost determines possible sample repolarization or MD formation outside the spot via boundary conditions, i.e. due to the surface charges $\sigma(x, y, z)$. Contrary, MD can not affect sufficiently on the $\sigma(x, y, z)$ value and distribution. Therefore it is worth to distinguish two boundaries of the transition layer 3 :

1) The inner boundary 23 , where $E_{p}=0$ allowing for intensity discontinuity, but $D_{3} \approx D_{s}, D_{2} \neq D_{s}$. Allowing for boundary conditions ( 3 ) and ( $9 b$ ) one can conclude that effective surface charge is the following: $\sigma\left(\mathbf{R}_{n}\right)=E_{p n} / 4 \pi$.

$\sigma\left(\mathbf{R}_{n}\right)=\frac{1}{4 \pi} \frac{G_{n} \Gamma}{e \mu_{e} s} n_{d}^{+}$
Really, it is obvious that this surface density is smaller than maximal value $D_{s} / 4 \pi$ (see $(9 \mathrm{c})$ ) necessary for the full spontaneous induction screening. The dependence of effective surface charge s over dimensionless intensity and impurity concentration is depicted in Fig. 2.

2) The outer boundary 31 , where spontaneous induction and field are much smaller than their bulk values due to the screening: $D_{3} \approx 0$ and $E \approx 0$. Allowing for boundary conditions ( 3 ) and (10a) one can conclude that $D_{1 n}=$ $=4 \pi \sigma$. Therefore the boundary conditions (3) acquire the form:

$D\left(r>>R_{n}\right)=D_{s},\left.D_{n}\right|_{\mathbf{r}=\mathbf{R} n}=4 \pi \sigma\left(\mathbf{R}_{n}\right)$.

Subject to the induction vector is parallel to $z$ axis and $\sigma E_{p n}>0$, it is obvious that $\sigma>0$ at $z<0$ and $\sigma<0$ at $z>0$ in the case when $G_{p z}$ is parallel to $z$ axis and vise versa (see Fig. 1).

Taking into consideration the free electrons concentration $\mathrm{n}_{\mathrm{e}}$ dependence on the donor concentration $n_{d}(9 \mathrm{~b})$, one can conclude from (10a) that $\sigma\left(\mathbf{R}_{n}\right)$ is proportional to $\sqrt{n_{d}^{0}}$ and $n_{d}^{0}$ for the large and small concentration values respectively. It is worth to underline that (9b) was derived in the case of constant $n_{d}^{0}$, but if transverse induction components and currents are much smaller than the longitudinal ones, $(10 \mathrm{a}, \mathrm{b})$ is valid for $n_{d}^{0}(x, y)$. Therefore, allowing for the fluctuations of donor concentration [13], the complete modulation of the it at distances of the cluster size order leads to the surface charge strongly inhomogeneous distribution of $\sigma(x, y)$. The dependence of effective surface charge s over dimensionless intensity is depicted in Fig. 2. Really, surface charges

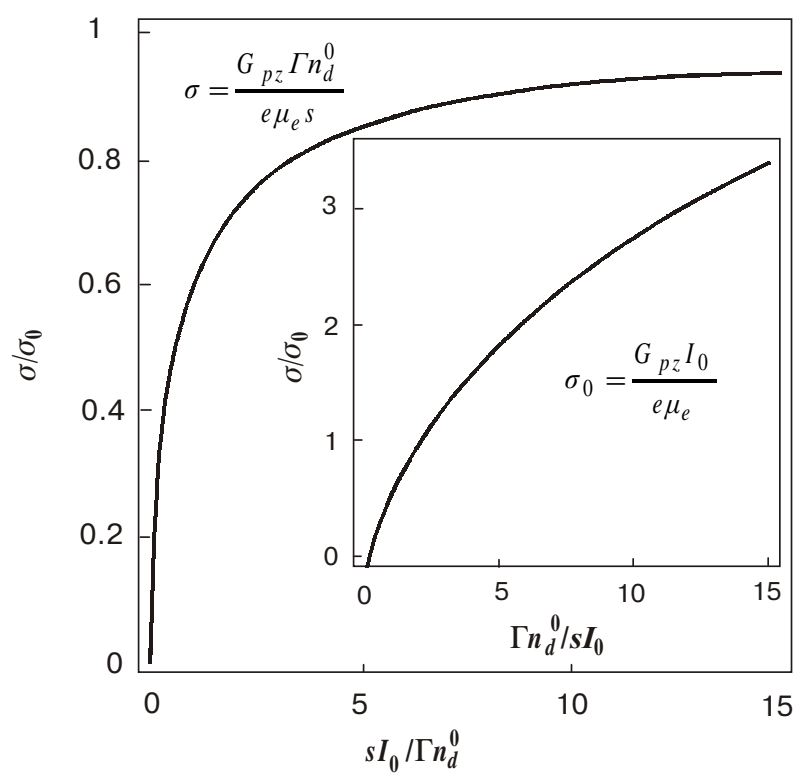

Fig. 2. The dependence of effective surface charge $\sigma$ over dimensionless intensity (basic plot) and dimensionless impurity concentration (inset). 


\section{A.N. Morozovska et al.: Photo-microdomains in ferroelectrics: formation ...}

are localized at the randomly distributed in the thin transition layer clusters of photoactive impurity with different sizes and averaged transverse period $b_{0}$. Each cluster can be treated as the seeding for one MD growth if only $D_{n} D_{s n}<0$, i.e. when $G_{p z}>0$ one obtains from (10b) that $\sigma>0$ at $z<0$ and $\sigma<0$ at $z>0$. So, the aforementioned light-induced MD formation would take place only in the samples with positive longitudinal component of Glass vector.

\section{Micro-domains formation}

\subsection{Light area with ideal flat edge and constant charge density}

At first let us consider the simplest case when the light area can be regarded infinite in $x, y$-directions with ideal boundaries at $z= \pm a_{0}$ and constant charge density $\sigma\left(-a_{0}\right)=\sigma_{0}, \sigma\left(a_{0}\right)=-\sigma_{0}$ (see Fig. 1 ), where $\mathrm{s}_{0}$ is the average value of the modulated charge density. Thus one can neglect transverse derivatives in equation (6), i.e $D_{z}=$ $=D(z)$. So, (6) and the boundary conditions (10b) acquire the form:

$$
\begin{gathered}
\alpha_{T} D+\beta D^{3}-\gamma_{L} \frac{d^{2}}{d z^{2}} D=0, \\
D\left(|z|>a_{0}\right)=D_{s}, \quad D\left(z= \pm a_{0}\right)=4 \pi \sigma_{0} .
\end{gathered}
$$

The novel one-dimensional kink solution [14] of (11a), which satisfy the boundary conditions, in the region $|z|>a_{0}$ can be rewritten as:

$$
\begin{gathered}
D=D_{s} \tanh \left[w\left(|z|-z_{0}\right)\right], \quad z_{0}=a_{0}+\frac{1}{w} \operatorname{arctanh}\left(\eta_{0}\right), \\
\eta_{0}=\frac{4 \pi \sigma_{0}}{D_{s}}, \quad D_{s}=\sqrt{-\frac{\alpha_{T}}{\beta}}, \quad w=\sqrt{-\frac{\alpha_{T}}{2 \gamma_{L}}}, \quad 0<\eta_{0}<1 .
\end{gathered}
$$

Substituting exact solution (11b) into (6) and performing integration over non-illuminated sample volume $V \approx$ $\approx S\left(l-a_{0}\right)$, one can obtain minimum free energy $F$ and its density $g=G / V$ values in the form:

$$
\begin{aligned}
& F_{\min }=V g_{B}+S g_{S} \underset{w \ell \gg>1}{\rightarrow} \\
& -\frac{\alpha_{T}^{2}}{4 \beta}\left(V-\frac{8 S}{w}\left(\frac{1}{3}+\frac{\eta_{0}}{2}-\frac{\eta_{0}^{3}}{6}\right)\right)
\end{aligned}
$$

Thus, free energy (11c) is the sum of bulk and surface energy densities $g_{B}$ and $g_{S}$ respectively. Solution (11b) is two symmetrical MD at $|z|<z_{0}$, where $D<0$, in the inverse polarized matrix at $|z|>z_{0}$, where $D>0$ (see solid curve in Fig. 2). Therefore the value $\left(z_{0}-a_{0}\right)$ is MD length $L_{M D}$. Notice, that as it follows from (11b), $L_{M D} \rightarrow \infty$ at $\eta_{0} \rightarrow$ 1, i.e. the repolarization of the matrix should take place at $\eta_{0}=1$. But it was shown above that this and higher values $\eta_{0}>1$ can not be achieved on the illuminated area boundary (see (9c) and (10a)).

Note, that solution (11b) becomes imaginary and has no meaning because of the finiteness of hyperbolic tangent function $(1 \geq|\tanh (z)|)$ for the case $4 \pi \sigma>D_{s}$. In this case one has to use another exact solution of the equation (11a) which does not oscillate and has the form $\pm D_{s} \operatorname{coth}\left(w\left(z-z_{0}\right)\right)$. It is obvious that this solution satisfy the boundary conditions for $4 \pi \sigma>D_{s}\left(\eta_{0}>1\right)$ because of the condition $|\operatorname{coth}(z)| \geq 1$. But this induction distribution diverges at $z=z_{0}$ and has infinite energy (8), therefore most probable that this distribution is unstable. In such a case real system could create strong electric fields at $z=z_{0}$, which would decrease s up to the case $4 \pi \sigma<D_{s}$ in a selfconsistent manner. Therefore, our theory also is not valid at $\eta_{0} \rightarrow 1$, owing to the strong nonlinear effects, which essentially decrease $\eta_{0}$ in a self-consistent manner. Using $\eta_{0}<<1$, for ideal flat light spot surface one obtains MD length $L_{M D}$ and domain wall thickness:

$$
L_{M D} \approx 4 \pi \sigma_{0} \frac{\sqrt{2 \gamma_{L} \beta}}{-\alpha_{T}}, \quad W_{M D} \sim \sqrt{-\frac{2 \gamma_{L}}{\alpha_{T}}}
$$

Notice, that as predicted in section 4.1, the sign of $\sigma_{0}$ determines MD-formation. Really, MD appear only in the case, when $D_{s} \sigma_{0}>0$.

\subsection{MD growth around one charged cluster}

At first let us consider the case when one charged cluster is localized at the light area boundary at $z=a_{0}$ and $|x|<b_{c}$ with negative charge density, $\sigma=-\sigma(x)$ (see inset to Fig. 3). Thus one cannot neglect transverse derivatives in equation (6), i.e $D_{z}=D(x, z)$. Neglecting the electric field transverse components, (6) and the boundary conditions (10b) acquire the form:

$\alpha_{T} D+\beta D^{3}-\left(\gamma_{L} \frac{d^{2}}{d z^{2}}+\gamma \frac{d^{2}}{d x^{2}}\right) D=0, \quad a_{0}<z$,
$D\left(z>>a_{0}\right)=D_{s}, \quad D\left(z=a_{0}\right)=-4 \pi \sigma(x)$.

About the features of $\sigma(x)$, we suppose the following. In the central part of the cluster charge density is maximum and rather homogeneous, i.e. $\sigma(x \approx 0)=\sigma_{c}$, but it sharply decreasing to zero approaching cluster edges, i.e. $\sigma\left(|x| \geq b_{c}\right)=0$. In order to obtain analytical expressions for spontaneous induction distribution, we propose the following representation for $\sigma(x)$ :

$\sigma(x)=\frac{D_{s}}{4 \pi} \theta\left(b_{c}-|x|\right) \tanh \left[\left(1-\frac{|x|}{b_{c}}\right) \operatorname{arctanh}\left(\frac{4 \pi \sigma_{c}}{D_{s}}\right)\right]$,

$0<\frac{4 \pi \sigma_{c}}{D_{s}}<1$

Where $\theta(x)$ is the step theta-function. The one-dimensional kink solution [14] of (13a), which satisfy the bound- 


\section{A.N. Morozovska et al.: Photo-microdomains in ferroelectrics: formation ...}

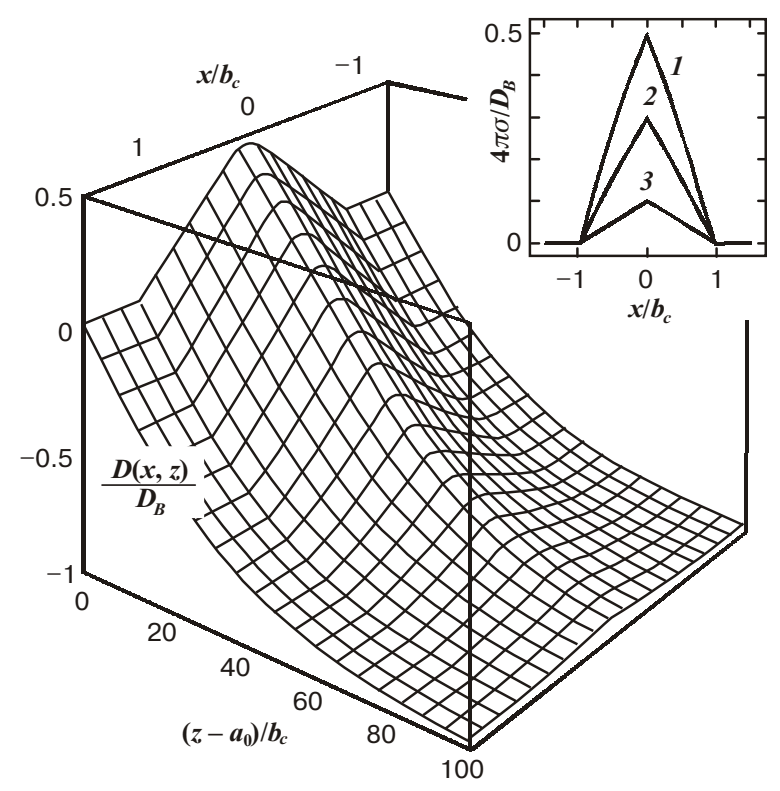

Fig. 3. Three dimensional plot for the dimensionless $D(x, z) / D_{s}$ distribution near one cluster for the following parameters $w_{c} b_{c}=$ $=0.022, \eta_{c}=0.5$. Inset represents $\mathrm{s}(\mathrm{x})$ distribution with the following parameter values $\eta_{c}=0.5,0.3,0.1$ for the curves $1,2,3$ respectively.

ary conditions with density (13b), in the region $z>a_{0}$ can be rewritten as:

$$
\begin{aligned}
& D=D_{s} \tanh \left[w_{c}\left(z-a_{0}\right)-\theta\left(b_{c}-|x|\right)\left(1-\frac{|x|}{b_{c}}\right) \operatorname{arctanh}\left(\eta_{c}\right)\right] \\
& z>a_{0}, \eta_{c}=\frac{4 \pi \sigma_{c}}{D_{s}}, D_{s}=\sqrt{-\frac{\alpha_{T}}{\beta}} \\
& w_{c}(x)=\sqrt{-\frac{\alpha_{T}}{2 \gamma_{L}}-\theta\left(b_{c}-|x|\right) \frac{\gamma}{\gamma_{L}}\left(\frac{\operatorname{arctanh}\left(\eta_{c}\right)}{b_{c}}\right)^{2}}
\end{aligned}
$$

Notice, that solution (14a) can be used as estimation at $x=0 ; \pm b_{c}$. It is easy to check from (14a) that our supposition $|e \varphi|<<k_{B} T$ used in (6) means $e D_{s} w_{c} R_{D}^{2}<<k_{B} T$. The region of repolarization, i.e. $\mathrm{MD}$, can be found from the condition $D<0$ (see $3 D$ plot in Fig. 3), so its outer boundary $D=0$ at $z=z_{0}(x)$ has the shape of the spike with the base at $z=a_{0}$ and $b_{c} \geq|x|$ :

$$
z_{0}(x)=a_{0}+\frac{\theta\left(b_{c}-|x|\right)}{w_{c}(x)}\left(1-\frac{|x|}{b_{c}}\right) \operatorname{arctanh}\left(\eta_{c}\right) .
$$

The end of the spike has the coordinates $x=0, z=$ $=z_{0}(0)$. Therefore the value $\left(z_{0}(0)-a_{0}\right)$ is maximum $\mathrm{MD}$ length $L_{\max }$. Note, that due to $w_{c} \leq w, L_{\max }>L_{M D}$ from (12a) always when $\sigma_{c}>\sigma_{0}$. Moreover, in the most cases the effective density $\sigma_{0}$ is much smaller than the peak one $\sigma_{c}$. Thus, one obtains:

$$
L_{\max }=\frac{1}{w_{c}(0)} \operatorname{arctanh}\left(\eta_{c}\right), \quad \frac{L_{\max }}{L_{M D}}=\frac{w \sigma_{c}}{w_{c}(0) \sigma_{0}}>>1
$$

The maximum transverse size of $\mathrm{MD} H_{\max }$ is the base $2 b_{c} \sim$ nm. So, from (14a) and (15a) one can obtain that when $w_{c} \rightarrow 0$

$$
\frac{L_{\max }}{H_{\max }} \rightarrow \infty \text { if only } \eta_{c} \rightarrow b_{c} \sqrt{\frac{-\alpha_{T}}{2 \gamma}} \text {. }
$$

The situation which corresponds to $(15 b)$ really means, that spike-like MD transforms into a very long needle-like one and in principle can intergrow through the perfect sample. In such a case it could be easily detected experimentally. The aforementioned intergrowth $(15 \mathrm{~b})$ is possible if maximum cluster charge density $\sigma_{c}$ is more that the critical one:

$\sigma_{c}>\sigma_{c r}$ where $\sigma_{c r}=\frac{D_{s}}{4 \pi} \tanh \left(b_{c} \sqrt{\frac{-\alpha_{T}}{2 \gamma}}\right)<\frac{D_{s}}{4 \pi}$.

One can estimate that at $\gamma \sim 10^{-14} \div 10^{-16} \mathrm{~cm}^{2}, \alpha_{T} \sim 10^{-2}$ (see, e.g. Ref. [15]), $b_{c} \sim 1 \mathrm{~nm}$, so $b_{c} \sqrt{-\alpha_{T} / 2 \gamma}<<1$ and

$$
\sigma_{c r} \approx \frac{D_{s}}{4 \pi} b_{c} \sqrt{\frac{-\alpha_{T}}{2 \gamma}}=\frac{3 \sqrt{3} b_{c}}{2 \sqrt{-2 \gamma \alpha_{T}}} E_{c}
$$

where $E_{c}$ is coercive electric field. One can estimate that the critical surface density $\sigma_{\max } \geq E_{c}$ as it should be expected. It is worth to underline that if $\sigma_{c}$ is defined, the intergrowth is possible for "small" clusters, namely:

$b_{c}<b_{c r}$,

$b_{c r}=\operatorname{arctanh}\left(\frac{4 \pi \sigma_{c}}{D_{s}}\right) \sqrt{\frac{2 \gamma}{-\alpha_{T}}} \approx$

$\approx \frac{4 \pi \sigma_{c}}{D_{s}} \sqrt{\frac{2 \gamma}{-\alpha_{T}}}<(1 \div 10) \mathrm{nm}$

Substituting exact solution (14a) into free energy functional (6) and integrating over $z$, then over $x, y$, we obtain minimum free energy $F$ and its density $g=F / V$ values in the same form and meaning that (11c) with substitution $\eta_{0} \rightarrow \eta_{c}$ and $w \rightarrow w_{c}$.

Notice, that in the case of radial symmetry $\sigma(x, y) \equiv$ $\equiv \sigma\left(r_{\perp}\right)$ and thus $D=D\left(r_{\perp}\right)$, we used solution (13b) for estimations after substitution $x \rightarrow r_{\perp}$. Really the aforementioned solution and all inequalities appeared to be a good approximation in the region where $\Delta_{\perp} \approx d^{2} / d r_{\perp}^{2}$, i.e. at $r_{\perp}>>b_{c} / \operatorname{arctanh}\left(\eta_{c}\right)$, i.e. not on the top of the MD spike. But the main conclusion is valid in case of radial symmetry: if $\sigma_{c}$ approaching $\sigma_{\max }$ determined by (16), one can observe conic needle-like MD intergrowing from charged clusters through the dark area of the PRC. So, exactly due to the transverse modulation effects the MD length can be sharply increased from the dozens of microns and up to the crystal length. 


\section{A.N. Morozovska et al.: Photo-microdomains in ferroelectrics: formation ...}

\subsection{Light area with modulated surface charge density}

Now let us consider the case when the cylindrical light area can be regarded plain near the polar axis $z$ with boundaries at $z= \pm a_{0}$ and inhomogeneous charge density $\sigma\left(-a_{0}\right)=\sigma(x, y), \sigma\left(a_{0}\right)=-\sigma(x, y)$, (charged circles in Fig. 1). About the features of $\sigma(x, y)$, we suppose the following:

$$
\begin{aligned}
& \langle\sigma\rangle=\sigma_{0},\left\langle\frac{d \sigma}{d x}\right\rangle=\left\langle\frac{d \sigma}{d y}\right\rangle=0, \\
& \left\langle\left(\frac{d \sigma}{d x}\right)^{2}\right\rangle=\left\langle\left(\frac{d \sigma}{d y}\right)^{2}\right\rangle=\left(\frac{\sigma_{0}}{b_{0}}\right)^{2} .
\end{aligned}
$$

The last expression in (17) means that the distribution function of $\sigma(x, y)$ spatial period is well-localized near average cluster period $b_{0}<<a_{0}$.

In is easy to verify by considering electrostatic problem, that the resulting electric field transverse components, generated by the numerous inhomogeneously distributed surface charges of one sign, are much smaller than the ones from single charge even at distances greater than average period $b_{0}$. So, after statistical averaging at $|z|>a_{0}$ the induction transverse components can be neglected. This statement confirms our initial supposition $\left|D_{x, y}\right|<<\left|D_{z}\right|$ (see commentary to (1b)). Thus one must take into account transverse derivatives in equation (6), i.e $D_{z}=D(x, y, z)$. So boundary conditions (9) acquire the form:

$$
D\left(|z|>>a_{0}\right)=D_{s}, \quad D\left(z= \pm a_{0}\right)=4 \pi \sigma(x, y) .
$$

The approximate analytical solution for $D$ can be found from minimum (6) by direct variational principle. This solution must be transformed into (11) if $\sigma(x, y)=\sigma_{0}$. Therefore we found it in the form analogous to precise one-dimensional solution (11), but satisfying the boundary conditions (18):

$$
\begin{aligned}
& D=D_{s} \tanh \left[w_{0}\left(|z|-z_{0}(x, y)\right)\right],|z|>a_{0}, \\
& z_{0}(x, y)=a_{0}+\frac{1}{w_{0}} \operatorname{arctanh}(\eta(x, y)), \\
& \eta(x, y)=\frac{4 \pi \sigma(x, y)}{D_{s}},\langle\eta(x, y)\rangle=\eta_{0}<<1, \\
& \left\langle\left(\frac{d \eta}{d x}\right)^{2}\right\rangle=\left\langle\left(\frac{d \eta}{d y}\right)^{2}\right\rangle=\left(\frac{\eta_{0}}{b_{0}}\right)^{2}, \\
& \eta_{0}=4 \pi \sigma_{0} \sqrt{-\frac{\beta}{\alpha_{T}}}, 0<\eta(x, y)<1 .
\end{aligned}
$$

Here the induction amplitude $D_{s}$ and domain wall thickness $1 / w_{0}$ are variational parameters.
Taking into account (17) one can integrate the free energy density (8) with the trial function (19) and elementary transformations [16] and easily obtain the free energy $\langle F\rangle$ dependence on the variational parameters. Taking into consideration, that $D_{s}$ is independent on $l$ in rather thick PRC and even coincides with $\pm \sqrt{-\alpha_{T} / \beta}$ in bulk ferroelectric, we obtain that it is possible if only $\partial\langle F\rangle / \partial D_{s}=0$ for arbitrary thickness $l$. In other words bulk and surface energies can be variated over $D_{s}$ independently. Therefore we obtain the following values for variational parameters and free energy minimum:

$$
\begin{aligned}
& D_{s}=\sqrt{-\frac{\alpha_{T}}{\beta}}, \quad w_{0}=\sqrt{-\frac{\alpha_{T}}{2 \gamma_{L}}-\frac{2 \gamma}{\gamma_{L}}\left(\frac{\eta_{0} / b_{0}}{1-\eta_{0}^{2}}\right)^{2}}, \\
& \langle G\rangle_{\min }=-\frac{\alpha_{T}^{2}}{4 \beta}\left(V-\frac{8 S}{w_{0}}\left(\frac{1}{3}+\frac{\eta_{0}}{2}-\frac{\eta_{0}^{3}}{6}\right)\right)
\end{aligned}
$$

Using (19) and (20), the $D$ distribution in the PRC is the following:

$$
\begin{aligned}
& D=\sqrt{-\frac{\alpha_{T}}{\beta}} \tanh \left[\sqrt{-\frac{\alpha_{T}}{2 \gamma_{L}}-\frac{2 \gamma}{\gamma_{L}}\left(\frac{\eta_{0} / b_{0}}{1-\eta_{0}^{2}}\right)^{2}}\left(|z|-z_{0}(x, y)\right)\right] \\
& |z|>a_{0}, \\
& z_{0}(x, y)=a_{0}+ \\
& +\operatorname{arctanh}\left(4 \pi \sigma(x, y) \sqrt{-\frac{\beta}{\alpha_{T}}}\right) / \sqrt{-\frac{\alpha_{T}}{2 \gamma_{L}}-\frac{2 \gamma}{\gamma_{L}}\left(\frac{\eta_{0} / b_{0}}{1-\eta_{0}^{2}}\right)^{2}} .
\end{aligned}
$$

Note, that at $\gamma \rightarrow 0$ the solution (21) coincides with (11) for flat spot edge, as it should be expected. Solution (21) is a plenty of MD at $a_{0}<|z|<z_{0}(x, y)$, where $D<0$, in the inverse polarized matrix at $|z|>z_{0}(x, y)$, where $D>0$. In accordance with (17) and (21), the mean MD length $\langle L\rangle=L_{M D}$, but the maximum $L_{\max }$ and minimum $L_{\text {min }}$ MD lengths are strongly different owing to the modulation of the surface density s, i.e. $\sigma \rightarrow 0$ when the donor concentration tends to zero and MD are absent near this kind of the surface. With the donor concentration $n_{d}^{0}$ increase the surface density increases as $\sqrt{n_{d}^{0}}$ and MD length also nonlinearly increases. Under the condition $4 \pi \sigma / D_{s} \rightarrow 1$ in the center of clusters MD length $L_{\max }$ tends to infinity, for the higher density values the strong depolarization field outside the illuminated area decreases $\sigma$. Therefore we can estimate the micro domain length as follows: 


\section{A.N. Morozovska et al.: Photo-microdomains in ferroelectrics: formation ...}

$$
\begin{aligned}
L_{\max } & >\frac{1}{\sqrt{-\frac{\alpha_{T}}{2 \gamma_{L}}-\frac{2 \gamma}{\gamma_{L}}\left(\frac{\eta_{0} / b_{0}}{1-\eta_{0}^{2}}\right)^{2}}}>L_{M D}, \\
L_{\min } & \approx 0 .
\end{aligned}
$$

The domain wall thickness $W_{M D} \sim 1 / w_{0}$ and mean MD thickness can be estimated as $H_{M D} \sim 2 b_{0}$. The shape of MD boundary is determined from the condition $D=0$ in the form $|z|=z_{0}(x, y)$. It is represented in Fig. 4 for three clusters with different maximal values $\sigma_{c}$ of the surface density $\sigma(x)=\sigma_{c} \sqrt{1-\left(r_{\perp} / b_{c}\right)^{2}}$ at the illuminated area edge. This density dependence on coordinates is obtained for the homogeneously charged spherical cluster with radius $b_{c}$. Value $\sigma_{c}$ is equal to the product of cluster diameter and volume charge density. It is seen that with $\sigma_{c}$ increase the MD length drastically increases.

The domain wall thickness $\sim 1 / w_{0}$ must be positive real number, so in the case $\eta_{0}<<1$ (see(19) and (20)) the critical average charge density $\sigma_{0}{ }^{c r}$ and critical cluster size (compare with (16)) exist:

$$
\sigma_{0}^{c r} \approx \frac{D_{s}}{4 \pi} b_{0} \sqrt{\frac{-\alpha_{T}}{4 \gamma}}, b_{0}^{c r} \approx \frac{4 \pi \sigma_{0}}{D_{s}} \sqrt{\frac{4 \gamma}{-\alpha_{T}}} .
$$

As it follows from (23), that when $\sigma_{0}$ tends to $\sigma_{0}{ }^{c r} \mathrm{MD}$ become very long and thin ones and in principle can intergrow through the perfect sample. In such a case they could be easily registered experimentally for example by optical methods. The aforementioned intergrowth is pos-

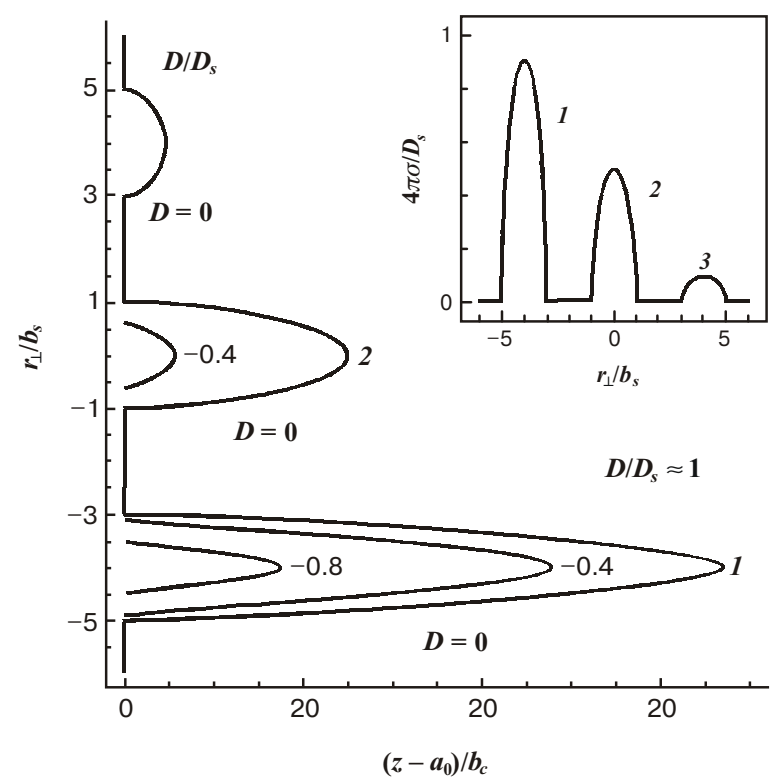

Fig. 4. The shapes of the MD boundary grown around the clusters with different $\sigma(x)$ distributions (see on the inset) with the following parameters: $w_{0} b_{0}=0.022$ and $4 \pi \sigma_{c} / D_{s}=0.9,0.5,0.1$ for the curves $1,2,3$ respectively. sible if the average cluster charge density $\sigma_{0}$ is more that the critical one (23). So, exactly due to the density modulation in transverse directions the MD length can be sharply increased.

\subsection{Comparison with the experiment on $\mathrm{LiNbO}_{3}$ : Fe crystals microdomain PILS}

It was shown earlier [17] that MD photo generation can take place not only at the surface but also in the volume of ferroelectric crystal. The rectangular specimens of the $\mathrm{LiNbO}_{3}$ monocrystal doped with $\mathrm{Fe}$ (the iron concentration in the melt was $\mathrm{N}[\mathrm{Fe}]=0.03 \div 0.05 \mathrm{wt}$. $\%$ ), faces transversal dimension $5-15 \mathrm{~mm}$ and thickness $2-5 \mathrm{~mm}$ was used in the experiment. Photoactivation is carried out by the narrow light beam with diameter $d_{L}=1.2 \mathrm{~mm}$, wavelength $\lambda_{L}=0.44 \mu \mathrm{m}$, intensity $P_{L}=20 \mathrm{~mW}$, which propagate along $\mathrm{x}$-axis perpendicularly to the polar axis and the crystal surface. This setup completely corresponds to the model geometry shown in Fig. 1. Investigation of the microdomains photo generation dynamics and its parameters determination is carried out by the method of testing light beam scattering, which propagates at small angle to the direction of $y$-axis. This scattering appeared as a weakly bent curve. The indicatrix of the scattering on MD dependence on the testing beam position in $x z$ plane allows one to determine the characteristics of defects arising in crystal under illumination.

As it was predicted in our model, scattering centers looks like needles, oriented along polar z-axis. The average MD length $L_{M D}=900 \mu \mathrm{m}( \pm 10 \%)$ is determined for the case, when the pump region has the view of the round cylinder with diameter $d_{L}=1.2 \mathrm{~mm}$, crossing the sample in the facet center normally to the crystal surface. The scattering indicatrix of the testing beam propagating close to the polar $z$-axis is measured for the determination of the average $\mathrm{MD}$ radius $b_{0}=0.9 \mu \mathrm{m}( \pm 10 \%)$.

These defects appear in the crystal volume only in the case when the pump intensity has the nonzero gradient along the polar axis $\partial I_{L} / \partial z \neq 0$. In particular, if the pump has the stripe form, oriented along $z$-axis, then MD do not arise in the crystal volume. This quite agrees with the proposed model, in accordance with which MD arise on the boundary of the illuminated region.

It should be noted that the disposition of MD relatively light boundary is asymmetric. In particular, MD penetration depth into illuminated region is approximately $1 / 3$ of their length. In accordance with our theory $\mathrm{MD}$ is situated outside the light spot in the case of sharp boundary light-shade. Diffuseness of this boundary leads to the charged layer thickness increase and, therefore, MD penetration to the illuminated region.

MD arise due to the seeding surface charge $\sigma$ on the light spot boundary, i.e. the main role of the pump is the spatial separation of charges in consequence of photo galvanic effect (PGE), the stimulation of MD arrange onset and the creation of the inner electric field with anomaly large voltage [12]. However, besides that, the pump beam can play the same role as the testing one. In 


\section{A.N. Morozovska et al.: Photo-microdomains in ferroelectrics: formation ...}

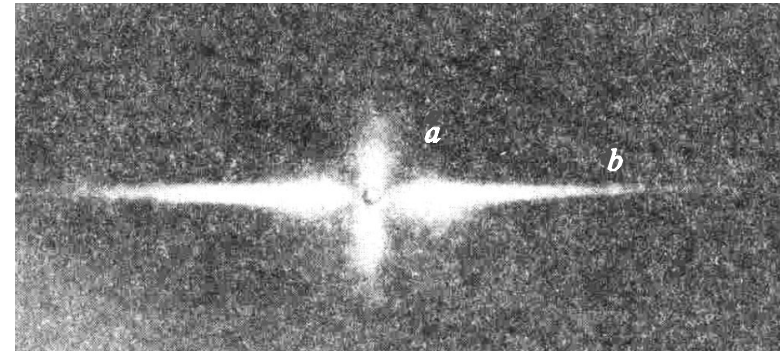

Fig. 5. The scattering of the pump beam in $\mathrm{LiNbO}_{3}$ : Fe. Letters " $a$ " and " $b$ " mark the photorefractive and photo-microdomain scattering respectively.

this case the typical scattering appears as a narrow strip oriented perpendicularly to z-axis (Fig. 5).

The density of MD, which defines average distance $\Delta l_{\perp}$ between them, was calculated from the testing radiation absorption coefficient value. Namely, the average MD quantity on the unit area of the surface, perpendicular to z-axis, is equal $N_{M D} \approx 7 \cdot 10^{5} \mathrm{~cm}^{-2}$. The quantity $\Delta l_{\perp}=12 \mu \mathrm{m}$ appears much less than both the average length $L_{M D}$ and the average radius $b_{0}$ of microdomains. This confirms the supposition used in our theory about the independence of individual microdomains.

\section{Conclusions}

The model of the MD formation in photorefractive uniaxial ferroelectrics near the illuminated area polarized due to the screening effects has been developed.

We have shown that MD onset does take place near the charged volume defects or clusters. Such clusters, as the photoinduced inhomogeneities at the points with the maximal photoactive impurity concentration, could create the surface charge density on the light spot edge.

When surface charges are localized at the nano-clusters of different size and charge density randomly distributed in the thin transition layer between light and dark, each cluster can be treated as the seeding for one MD growth. Therefore the numerous MD appear around the illuminated area.

We obtained, that when the cluster charge density is more that the critical one, MD become very long and thin ones and in principle can intergrow through the perfect sample. In such a case they could be easily registered experimentally. So, exactly due to the transverse modulation effects the MD length can be sharply increased from the dozens of microns and up to the crystal length.
The considered light-induced MD formation would take place only in the samples with positive longitudinal component of Glass vector.

The aforementioned mechanism of MD formation, MD shape and sizes are in a good qualitative agreement with the experiments on MD observation [5], [17].

\section{References}

1. P. Lehnen, W. Kleemann, Th. Woike, R. Pankrath, Linear birefringence studies of spontaneous and precursor polarization // Eur. Phys. J., B14, pp. 633 (2000).

2. L.I. Ivleva, T.R. Volk, P. Lykov, N. Polokov, D. Isakov, M. Wöhlecke, Modification of photorefractive properties of SBN: Ce crystals by means of co-doping La impurity // Appl. Phys. Lett., 79, pp. 854 (2001).

3. T.R. Volk, Th. Woike, U. Dörfler, R. Pankrath, L.I. Ivleva, M. Wöhlecke, Ferroelectric hysteresis phenomena on holographic properties of SBN crystals doped with RE elements // Ferroelectrics., 202, pp. 53 (1997).

4. V.M. Fridkin, Photoferroelectrics (Photosegnetoelectriki), Nauka, Moscow (1979) (in Russian).

5. T. Granzow, U. Dörfler, Th. Woike, M. Imlau, R. Pankrath, M. Wöhlecke, W. Kleemann, Influence of pinning-effects on the ferroelectric hysteresis in cerium-doped strontiumbarium-niobate // Phys. Rev., B63, pp. 17401 (2001).

6. G.M. Guro, I.I. Ivanchik and N.F. Kovtonyuk, Semiconductor properties of barium titanate // Sov. Phys. Solid State 10, pp. 100 (1968) (in English) [Phys. Tverd. Tela 10, pp. 134 (1968) (in Russian)].

7. N.A. Pertsev, A.G. Zembilgotov and A.K. Tagantsev, Effect of mechanical boundary conditions on phase diagrams of epitaxial ferroelectric thin films // Phys. Rev. Lett., 80, pp. 1988 (1997)

8. W. Cao and L.E. Cross, Theory of tetragonal twin structures in ferroelectric perovskites with a first-order phase transition // Phys. Rev., B 44, pp. 5 (1991).

9. U.S. Kuzminov, Litium niobate and tantalate are the materials for nonlinear optics, M. Nauka (1975) (in Russian).

10. N.V. Kukhtarev, V.B. Markov, S.G. Odulov, M.M. Soskin, V.L. Vinetskii, Holographic storage in electro-optic crystals // Ferroelectrics, 22, pp. 949-960 (1979).

11. H. Haken, Synergetics, Springer-Verlag, Berlin (1978)

12. V.M. Fridkin, Ferroelectrics-Semiconductors (Segnetoelectrikipoluprovodniki), Nauka, Moscow (1976) (in Russian).

13. Kh.S. Bagdasarov, E.M. Uyukin, V.A. Meleshina, A.G. Yasparyan, Detection of inhomogeneities in structures of lithium niobate // Journal of Crystal Growth, 52, pp. 983 (1981).

14. R.K. Dodd, J.C. Eilbeck, J.D. Gibbon and H.C. Morris, Solitons and Nonlinear Wave Equations, Academic Press Inc., London (1984).

15. M.E. Lines and A.M. Glass, Principles and Applications of Ferroelectrics and Related Phenomena, Oxford University Press (1978).

16. H.B. Dwight, Tables of Integrals, The Macmillan Company, New York (1961)

17. V.V. Lemeshko, V.V. Obukhovsky and A.V. Stoyanov, Photogeneration needle-like domains in lithium niobate crystals // Fiz. Tv. Tela, 34, pp. 1833-1838 (1992). 